\title{
Quantitative imaging of sodium concentrations in soil-root systems using magnetic resonance imaging (MRI)
}

\author{
Adi Perelman • Naftali Lazarovitch • Jan \\ Vanderborght • Andreas Pohlmeier (D)
}

Received: 11 May 2020 / Accepted: 28 June 2020 /Published online: 25 July 2020

(C) The Author(s) 2020

\begin{abstract}
Aims Demonstrating the potential of MRI as a 3D, noninvasive and continuous measurement technique to map $\mathrm{Na}^{+}$concentration distributions in soil and around roots. Methods Dissolved $\mathrm{NaCl}$ in soil and soil-plant systems was mapped by $3 \mathrm{D}{ }^{23} \mathrm{Na}$-MRI. The lower limit of detectability in saturated and unsaturated porous media was evaluated, followed by evaporation experiments to test the quantification. Finally, $\mathrm{Na}^{+}$enrichment around tomato roots, irrigated with saline solution under low/ high transpiration rates (LT, HT), was imaged in parallel to the root system,.
\end{abstract}

Responsible Editor: Peter J. Gregory

\footnotetext{
A. Perelman · N. Lazarovitch

French Associates Institute for Agriculture and Biotechnology of Drylands, Jacob Blaustein Institutes for Desert Research,

Ben-Gurion University of the Negev, Midreshet Ben-Gurion, Sede Boqer Campus, 849900, Israel

J. Vanderborght

Department of Earth and Environmental Sciences, Faculty of

Bioscience Engineering, KU Leuven, Leuven, Belgium

J. Vanderborght

Institute of Bio- and Geoscience, Agrosphere Institute, IBG-3, Forschungszentrum Jülich GmbH, Jülich, Germany

e-mail: a.pohlmeier@fz-juelich.de
}

Present Address:

A. Pohlmeier $(\triangle)$

Institute of Bio- and Geoscience, Agrosphere Institute, IBG-3,

Forschungszentrum Jülich GmbH, Jülich, Germany

e-mail: a.pohlmeier@fz-juelich.de
Results A spin echo pulse sequence allowed the quantitative mapping of the volume concentration of $\mathrm{NaCl}$ in sandy porous medium. Evaporation experiments showed slight enrichment in the top surface layer, plus uniform temporal enrichment in the deeper layers. In the tomato experiments, enrichment was more distinct under HT than under LT. Concentration-distance correlation curves revealed thin enrichment zones ranging a few mm around the roots.

Conclusions MRI can map $\mathrm{Na}^{+}$non-invasively in $3 \mathrm{D}$ at relevant concentrations for root activity. Visualizing water content, roots and $\mathrm{Na}^{+}$on the same scale is possible, despite limitations of different scanning times and resolution. This opens a route for further quantitative investigations of salt enrichment processes in soil and soil-plant systems.

Keywords $\mathrm{Na}^{+}$accumulation $\cdot \mathrm{Na}^{+}$distribution $\cdot$ Roots and soil $\cdot$ Saline irrigation $\cdot \mathrm{NMR} \cdot{ }^{23} \mathrm{Na}-\mathrm{MRI}$

\section{Introduction}

Salinity is a major environmental factor limiting agricultural crops productivity, since most crops are sensitive to salinity stress caused by high salt concentrations in the soil (Rodriguez et al. 1997). When plants transpire, water moves from the soil to the roots and then to the transpiring leaves along pressure gradients (Denmead and Shaw 1962). Moreover, there is a correlation between the amount of water transpired and mineral uptake from the soil (Barber 1962) so that $\mathrm{Na}^{+}$ 
movement toward the root will also be affected by transpiration rate. On the other hand, semipermeable properties of the root surface lead to accumulation of salt outside the root and give rise to an additional osmotic potential, impeding water uptake. Thus, the interplay between transpiration rate, osmotic potential and the ability of roots to extract water from saline soil often limits growth and development of plants (Rodriguez et al. 1997). Previous studies have reported differences in $\mathrm{Na}^{+}$accumulation around roots depending on transpiration rate, with higher $\mathrm{Na}^{+}$concentrations around the roots compared with the bulk soil for higher transpiration rates (Riley and Barber 1970; Sinha and Singh 1974).

The sensitive nature of roots in soil often requires special equipment and procedures for their studies (Polomsky and Kuhn 2002). This applies in particular to the investigation of the root-soil interface. Moreover, studies aiming to quantify root growth in soils are often limited by the lack of suitable methods for time-lapse, non-destructive measurements (Kuchenbuch and Ingram 2002). In today's root research, there is therefore a growing need to develop new imaging methods that allow simultaneous, non-invasive mapping of root growth and architecture in combination with a quantitative mapping of the distribution of water and dissolved substances in soils.

Various root studying platforms have been developed to track root growth non-invasively, hydroponics, aeroponics, agar and germination paper (e.g., Hund et al. 2009). Nevertheless, some are limited to primary growth only and methods like hydroponics and aeroponics are not suitable for measuring processes at the root interface, like solute gradients. A solution to these problems can be using a paper-based growth system such as rhizoslides (Jorda et al. 2018; Le Marié et al. 2014; Perelman et al. 2020), as they provide a relatively simple access to solute gradients around roots. However, it is impossible to track root development in three dimensions (3D) as the root system grows on top of the paper. In contrast, quantitative imaging of $3 \mathrm{D}$ root traits in soil can help achieving better mechanistic understanding of root system architecture (RSA) as it develops in the natural soil environment (Metzner et al. 2015). X-ray-computed tomography (X-ray CT) is becoming a common method for 3D imaging of roots (Flavel et al. 2012; Koebernick et al. 2014; Mairhofer et al. 2015), but, in contrast to MRI, is unsuitable to detect dissolved $\mathrm{NaCl}$ at low concentrations. Although $\mathrm{NaCl}$ was used occasionally as contrast agent in medical
X-ray-CT (Billich et al. 2008), the concentrations of such $\mathrm{NaCl}$ solutions are lethal for plants and therefore inconvenient for plant studies. On the other hand, magnetic resonance imaging (MRI) offers the potential of imaging root system architecture, processes at the rootsoil interface, and $\mathrm{NaCl}$ distribution at low concentrations non-invasively and in 3D (Olt et al. 2000).

MRI is a convenient imaging technique for noninvasive 3D imaging of subsurface soil processes in real time and time dependent. It is best known from medical diagnostics where it has been regularly used for over 30 years. Since then, applications of MRI for imaging structures and processes in geo- and material-sciences have become more and more common. Its advantages are its non-invasiveness, its sensitivity to water or some dissolved ions, and that it offers a many ways of specifying the signal information by variation of experimental parameters.

NMR microimaging methods have been developed for applications in material and geosciences (Blümich 2000; Blümich et al. 2014; Callaghan 1991; Neu et al. 2010), but also for the imaging of root-soil processes (Haber-Pohlmeier et al. 2019; Haber-Pohlmeier et al. 2018). They typically use vertical magnets with bore sizes of only some centimeters and allow finer resolution and higher sensitivity for smaller specimens such as plants or soil columns, although the sample size is limited (Haber-Pohlmeier et al. 2019; Van As 2007). The use of MRI in environmental sciences is not restricted to water but also allows the examination of hydrocarbons or other NMR active nuclei such as ${ }^{23} \mathrm{Na},{ }^{19} \mathrm{~F}$, and others (Rokitta et al. 2004; Simpson et al. 2007). In this context the imaging of $\mathrm{Na}^{+}$in porous media is of high interest especially due to the high ecological and economical importance of salinization processes in soils (Hadrich 2011; Welle and Mauter 2017).

Sodium MRI in porous media has some special problems, making it somewhat more difficult to use than hydrogen nucleus MRI. The quadrupole magnetic moment of ${ }^{23} \mathrm{Na}$ and the smaller gyromagnetic ratio cause significantly lower sensitivity and enhanced signal decay, i.e. relaxation (Kausik et al. 2018; Mitchell and Fordham 2017). The stronger contribution of bulk relaxation compared to surface relaxation implies that the relaxation acceleration of $\mathrm{Na}^{+}$in porous media is only observed for pore sizes smaller than $1 \mu \mathrm{m}$ (Rijners et al. 2004). During the last decade, several groups have started using MRI for studying $\mathrm{Na}^{+}$transport and crystallization in inorganic building materials (Pel et al. 
2016; Rijners et al. 2004) or food (Veliyulin et al. 2009), but it has also been applied to plant sciences. For example, Rokitta et al. (2004) and Olt et al. (2000) used ${ }^{23} \mathrm{Na}^{+}$ microimaging for studying physiological adaptations of salt tolerant plants by monitoring the $\mathrm{Na}^{+}$distribution in living plants and characterizing the tissue using relaxation time mapping. Nevertheless, none of these studies monitored changes of $\mathrm{Na}^{+}$distribution in the soil and its accumulation at the root-soil interface as a function of the plant water uptake and transpiration rate. Therefore, the potential of ${ }^{23} \mathrm{Na}$ MRI for investigation of soil samples should be explored in more detail.

This conceptual study intends to demonstrate the potential of MRI as a 3D, non-invasive, nondestructive and time lapse measurement technique that quantifies spatio-temporal dissolved $\mathrm{Na}^{+}$concentration distributions in soil and around roots. The general objective was the non-invasive $3 \mathrm{D}$ monitoring of $\mathrm{Na}^{+}$ distribution in soil and around roots by MRI, which, to our knowledge, has not yet been carried out. First, the NMR relaxation properties of the ${ }^{23} \mathrm{Na}$ signal in sandy soil were determined since these properties are the basis for any NMR image interpretation. Part of this step was also the verification of proportionality of the observed NMR echo intensity to the volume concentration of dissolved $\mathrm{Na}^{+}$. In the second step, we monitored the evaporation driven $\mathrm{NaCl}$ accumulation at the soil surface in bare sand by MRI. The quantitative imaging of $\mathrm{Na}^{+}$in soils by MRI was evaluated by comparing the total Na inflow with the amount determined in the MRI images. The third step comprised a study of the $\mathrm{Na}^{+}$ accumulation patterns in soil columns during water uptake by tomato root systems under high and low transpiration conditions. The $\mathrm{Na}^{+}$concentration was imaged with ${ }^{23} \mathrm{Na}$-MRI and the roots with ${ }^{1} \mathrm{H}$-MRI. The co-registered $\mathrm{Na}$ and root images were used to assess $\mathrm{Na}$ accumulation patterns around roots for different transpiration rates.

\section{Materials and methods}

MRI principles MRI is based on the quantum mechanical effect of nuclear magnetic resonance, NMR. Nuclear magnetism results from the nuclear spin of several atomic nuclei such as ${ }^{1} \mathrm{H},{ }^{23} \mathrm{Na},{ }^{19} \mathrm{~F}$, and ${ }^{13} \mathrm{C}$ and the linked microscopic magnetic moments. Among these, the hydrogen atom nucleus with a spin of $1 / 2$ is most widely used due to its high abundance in many organic or inorganic systems in form of water, hydrocarbons, or lipids and its high NMR sensitivity. The spins interact with an external magnetic field $\mathbf{B}_{0}$ by alignment of their projection either parallel or antiparallel to its direction $\mathbf{z}$ with given energy levels. Where $\gamma$ is the gyromagnetic ratio of the nucleus under consideration. The energy levels, two in case of ${ }^{1} \mathrm{H}$, which are separated by an energy of $\hbar \gamma\left|\mathbf{B}_{0}\right|$, are populated according to Boltzmann's law resulting in an observable macroscopic magnetization for an ensemble of spins. This magnetization can interact with external $r f$-radiation if it is in resonance with the Larmor frequency $\gamma_{\mathrm{L}}=\gamma_{\mathrm{H}}\left|\mathbf{B}_{0}\right| / 2 \pi$. In most cases, the excitation radiation is applied in form of a pulse with a length of some microseconds to few milliseconds. It results in a precession motion of the magnetization around $\mathbf{z}$ with the Larmor frequency which is detected by the receiver system and creates a signal $S_{0}$. Immediately after excitation, the precessing magnetization decays with two relaxation processes termed as $T_{1}$ and $T_{2}$. For the sake of simplicity, we restrict on NMR of liquids in the following, for details please refer to the literature (Blümich 2000; Blümich et al. 2014; Callaghan 1991; McRobbie et al. 2007). The relaxation times as well as the signal depend on the water content of the sample, the interaction of the liquid with its chemical and physical surroundings, and of the local diffusional and translational motion.

The inverse relaxation times are composed of different terms describing bulk relaxation, surface relaxation, and, in case of $T_{2}$, relaxation due to diffusion in magnetic field gradients. In porous media one expects following relations (Coates et al. 1999):

$$
\begin{aligned}
1 / T_{1} & =1 / T_{1, \text { bulk }}+\rho_{1} S / V \text { and } 1 / T_{2, \text { eff }} \\
& =1 / T_{2, \text { bulk }}+\rho_{2} S / V+\left[1 / T_{2, \text { diff }}\right]
\end{aligned}
$$

where the subscript indices refer either to longitudinal $\left(T_{1}\right)$ or transverse $\left(T_{2}\right)$ relaxation. The bulk relaxation terms describe unrestricted motion of liquids or dissolved ions in solution. The influence of faster relaxation at the pore walls is incorporated by specific surface relaxivity parameters $\rho_{1}$ and $\rho_{2}$ and the pore surface to volume ratio $S / V$. The additional term in square brackets for $T_{2}$ relaxation describes the acceleration of relaxation due to diffusion in internal magnetic field gradients.

Imaging The precessing magnetization can be manipulated by additional switching of magnetic field gradients plus additional rf-pulses. In magnetic resonance 
imaging, MRI, magnetic field gradients are applied in the Cartesian directions, $\mathbf{x}, \mathbf{y}$, and $\mathbf{z}$ to spatially encode the signal, for details please refer to the literature, e.g. (McRobbie et al. 2007). Finally, the image is obtained by Fourier transformation of the raw signal, which is recorded in the time domain. It can be either a multislice or a 3D image. Please note that this is one fundamental difference to other important 3D imaging methods such as X-ray-CT and neutron-CT, where projection images are monitored in real space. Furthermore, the MRI signal relies on the interaction of the excited spin ensemble with the local dynamics of the liquid in the porous system or the biological tissue, whereas for $\mathrm{X}$-ray- and neutron-CT the interaction is the attenuation of the primary beam.

In MRI, the entire combination of magnetic field gradients and $\mathrm{rf}$ pulses is termed as pulse sequence. The contrast relies on the interplay of the physical properties of the system with the type of pulse sequence and its parameters. Thus, there are many pulse sequences available, which are set up for special purposes. In general, liquid content, relaxation times or diffusion effects weight the contrast. Moreover, physical parameters such as spin density (volume concentration of the nuclei under consideration such as ${ }^{1} \mathrm{H}$ or ${ }^{23} \mathrm{Na}$, e.g. water or $\mathrm{NaCl}$ content), relaxation times, diffusion coefficients, or flow velocities can be mapped directly. This is possible either by adequate setting of parameters (see below) or by application of additional filters prior to the imaging part of the sequence.

The working horse of MRI in porous media is the socalled spin echo pulse sequence. The spatially resolved voxel signal $S_{\mathrm{x}, \mathrm{y}, \mathrm{z}}$ obeys the following relation assuming a $90^{\circ}$ excitation $r f$-pulse:

$S_{x, y, z}=S_{0, x, y, z}\left(1-\exp \left(-\frac{t_{R}}{T_{1}}\right)\right) \exp \left(-\frac{t_{E}}{T_{2, e f f}}\right)$

where $S_{0, \mathrm{x}, \mathrm{y}, \mathrm{z}}$ is proportional to the spin density, i.e. the number of ${ }^{1} \mathrm{H}$ or ${ }^{23} \mathrm{Na}^{+}$atoms or ions, respectively, per volume. The repetition time $t_{\mathrm{R}}$ and the echo time $t_{\mathrm{E}}$ are set by the operator. If the repetition time $t_{\mathrm{R}}$ is significantly longer than the longitudinal relaxation time $T_{1}$ and if the echo time $t_{\mathrm{E}}$ is significantly shorter than the effective transverse relaxation time $T_{2 \text {,eff }}$, the signal $S_{\mathrm{x}, \mathrm{y}, \mathrm{z}}$ is proportional to the water or sodium content in the respective voxel. For this reason, one should determine the transverse and longitudinal relaxation times prior to imaging experiments. In practice, it is possible to tune the pulse sequence to be sensitive for the spin density (water or $\mathrm{NaCl}$ content) by setting $t_{\mathrm{E}}>>T_{2 \text {,eff }}$ and $t_{\mathrm{R}}>5 \times T_{1}$ in Eq. 2.

MRI experiments All experiments were conducted in a Bruker super wide bore (SWB) scanner with a field strength of $4.7 \mathrm{~T}$ corresponding to Larmor frequencies of $52.9 \mathrm{MHz}$ for ${ }^{23} \mathrm{Na}$ and $200 \mathrm{MHz}$ for ${ }^{1} \mathrm{H}$. The system was equipped with a $600 \mathrm{mT} /$ $\mathrm{m}$ gradient system. Two $40 \mathrm{~mm}$ and $66 \mathrm{~mm}$ birdcage resonators were available for imaging of ${ }^{23} \mathrm{Na}$ and ${ }^{1} \mathrm{H}$, respectively. An insertable mini gradient system with a maximum strength of $1.4 \mathrm{~T} / \mathrm{m}$ and $25 \mathrm{~mm}$ birdcage resonators for ${ }^{23} \mathrm{Na}$ and ${ }^{1} \mathrm{H}$ allowed higher resolution. All scans were controlled by a Bruker Avance III console and Paravision software (Bruker, Rheinstetten, Gemany). ${ }^{23} \mathrm{Na}$ imaging was performed by a $3 \mathrm{D}$ spin echo sequence with an echo time $t_{\mathrm{E}}=2.6 \mathrm{~ms}$, an acquisition bandwidth $b w=$ $50 \mathrm{kHz}$, and a repetition time $t_{\mathrm{R}}=0.3 \mathrm{~s}$. Further important parameters of the imaging experiments are summarized in Table 1. With the given field-of view $(\mathrm{FoV})$ and image matrix sizes we could obtain typical resolutions of $1 \mathrm{~mm}$. NMR relaxation times were measured additionally for diluted $0.2 \mathrm{~mol} / \mathrm{L}$ $\mathrm{NaCl}$ solutions in sand by CPMG and inversionrecovery (IR) sequences with following parameters. The echo times ranged from $t_{\mathrm{E}}=0.5 \mathrm{~ms}$ to $4.0 \mathrm{~ms}$, the acquisition bandwidth was $b w=50 \mathrm{kHz}$, and the repetition time was $t_{\mathrm{R}}=0.3 \mathrm{~s}$. The obtained relaxation curves were analyzed by inverse Laplace transformation to calculate relaxation time spectra (Prospa software, Magritek, New Zealand).

Calibration The calibration sample employed in this study was composed of a bundle of four glass tubes with $15 \mathrm{~mm}$ inner diameter and a porous plate as bottom for wetting and desiccation. Each tube was filled with sand (FH31, Quarzwerke Frechen, Gemany) with a packing density of $1.6 \mathrm{~g} / \mathrm{cm}^{3}$ and saturated from bottom with $\mathrm{NaCl}$ solutions of $0.04,0.1,0.2$ and $0.4 \mathrm{~mol} / \mathrm{L}$ to a volumetric water content of $\theta=0.36 \mathrm{~cm}^{3} / \mathrm{cm}^{3}$. Subsequent degrees of water contents of $\theta=0.30 \mathrm{~cm}^{3} / \mathrm{cm}^{3}$, $0.25 \mathrm{~cm}^{3} / \mathrm{cm}^{3}$ and $0.16 \mathrm{~cm}^{3} / \mathrm{cm}^{3}$ were adjusted by pressing the tubes on filter papers for desiccation. The bundle was scanned by the $40 \mathrm{~mm}$ resonator at the given saturation degrees using a 3D spin echo sequence, see Table 1. 
Evaporation The $\mathrm{NaCl}$ accumulation in a sand packing was imaged in a Perspex column of $30 \mathrm{~mm}$ inner diameter filled with sand (FH31, Quarzwerke Frechen, Gemany) to a height of $50 \mathrm{~mm}$ so that the entire sample fitted well into the irradiated volume of the $40 \mathrm{~mm}{ }^{23} \mathrm{Na}$ resonator (Fig. 1c). The column was closed at the bottom by a porous plate with an air entry value of $-70 \mathrm{~cm}$ and connected to a Mariotte bottle filled with $0.02 \mathrm{M}$ and $0.03 \mathrm{M} \mathrm{NaCl}$ solutions. It was placed on a balance outside the magnet to monitor solution loss due to evaporation and could be vertically shifted. Thus we adjusted steady-state water contents corresponding to hydraulic heads of $-10 \mathrm{~cm}$ and $-20 \mathrm{~cm}$ for the two evaporation series. The evaporation was started by guiding a stream of $120 \mathrm{~L} / \mathrm{h}$ dry $\mathrm{N}_{2}$ gas $2 \mathrm{~cm}$ above the surface of the sand packing inside the magnet and monitored the ${ }^{23} \mathrm{Na}$ for 8 days. Finally, we scanned the water content distribution by replacing the column into the $66 \mathrm{~mm}{ }^{1} \mathrm{H}$ resonator without disconnection from the Mariotte bottle so that the hydraulic head remained unchanged. Table 1 summarizes the pulse sequence details.

Accumulation in tomato root systems Tomato plants were grown in glass cuvettes $(20 \mathrm{~mm}$ diameter, $100 \mathrm{~mm}$ height), filled with FH31 sand up to ca. $80 \mathrm{~mm}$ (Fig. 1d) and equipped with a $5 \mathrm{~mm}$ wide marker tube containing $0.05 \mathrm{~mol} / \mathrm{L} \mathrm{NaCl}$ for calibration and positioning. Plants were irrigated with saline water $\left(\mathrm{EC}=4.5 \mathrm{dSm}^{-1}\right.$ or $\left.0.0416 \mathrm{~mol} / \mathrm{L}\right)$ and were grown under two transpiration rates, high and low (see section 2.1). The low transpiration rate was adjusted by putting the plant into a desiccator so that the relative humidity was close to $100 \%$, whereas for the high transpiration rate the plant remained in the air-conditioned room at a relative humidity of $60 \%$. The average transpiration rates were $0.54 \mathrm{~cm} /$ day and $0.3 \mathrm{~cm} /$ day, the temperature was $23^{\circ} \mathrm{C}$, and the illumination strength was $400 \mu \mathrm{mol} /\left(\mathrm{m}^{2} \mathrm{~s}\right)$ for $12 \mathrm{~h}$ a day. The cuvettes were irrigated from the bottom by capillary rise in a small container. Six weeks after seeding, each plant was scanned twice: firstly, with the ${ }^{1} \mathrm{H}$-coil for imaging the RSA, and secondly with the ${ }^{23} \mathrm{Na}$-sensitive coil for mapping the $\mathrm{Na}^{+}$distribution. The samples were positioned in the magnet's bore at a temperature of $18{ }^{\circ} \mathrm{C}$ and in the dark so that the plants were barely transpiring. These conditions allowed long measurement times without dehydration or significant changes in the soil water content. The entire column was not scanned since it would require three different scans at three depths, which would have been too time consuming. The duration of a single scans and their FoV are included in Table 1.

Image processing The ${ }^{1} \mathrm{H}$ images representing the RSA were processed without filtering by segmentation of the roots from the surrounding soil material using the region growing tool implemented in the image processing software Fiji (Schindelin et al. 2012) and subsequent binarization. Finally, a 2D Euclidean distance transformation was performed on all slices of the binary RSA images for later correlation with the $\mathrm{Na}^{+}$concentrations using IDL (Harris Geospatial Solutions, Inc., Broomfield, CO). The ${ }^{23} \mathrm{Na}$ images were sine-filtered before inverse Fourier transformation to remove random noise outside the cuvettes. The sodium concentration maps were calculated next by normalization of the intensities of the ${ }^{23} \mathrm{Na}$ images on the intensity of the built-in marker tube, filled with defined $\mathrm{NaCl}$ solution. In the next step, the sodium concentration maps, and the RSA images were co-registered manually to match resolution and orientation by translation and rotation operations. This was followed by the correction of systematic vertical signal intensity inhomogeneities with help of the marker tube containing a solution of $0.05 \mathrm{~mol} / \mathrm{L} \mathrm{NaCl}$. Finally, $\mathrm{Na}^{+}$ concentration maps were obtained by normalization on the marker tubes. The correlation diagrams between $\mathrm{Na}^{+}$concentration and distance to the nearest root were obtained analogous to the procedure used for volumetric water content distance maps. First, the co-registered $\mathrm{Na}^{+}$images were scaled to the resolution of the RSA images. Second, the data were collected on Cartesian grid coordinates together with the respective distance values, obtained from the distance transform map of the RSA image and the $\mathrm{Na}^{+}$concentrations. Thus, the resulting 5 column matrix was composed of the three Cartesian grid coordinates, the distance to the nearest root and the $\mathrm{Na}$ concentration. A plot of the concentration in each voxel vs its distance to the nearest root is termed "concentration distance correlation diagram". Figure 10 illustrates the procedure in the next section. All image processing steps were performed with Fiji (Schindelin et al. 2012) or IDL. 
Table 1 parameters of the different imaging experiments

\begin{tabular}{|c|c|c|c|c|c|c|c|c|c|}
\hline Sample & nucl. & coil & sequence ${ }^{*}$ & FoV $\left(\mathrm{cm}^{3}\right)$ & matrix & $\mathrm{N}_{\mathrm{E}}^{\#}$ & time (h) & $\mathrm{N}_{\mathrm{av}}^{+}$ & $\theta\left(\mathrm{cm}^{3} / \mathrm{cm}^{3}\right)^{\S}$ \\
\hline $\begin{array}{l}\text { calibration } \\
\text { experiment }\end{array}$ & ${ }^{23} \mathrm{Na}$ & large & 3D MSME & $4.8 \times 4.8 \times 4.8$ & $48 \times 48 \times 24$ & 4 & 12.3 & 128 & $0.36,0.30,0.25,0.15$ \\
\hline Evaporation samples & ${ }^{23} \mathrm{Na}$ & $\begin{array}{l}\text { large } \\
\text { large }\end{array}$ & $\begin{array}{l}\text { 3D RARE } \\
\text { 2D MSME }\end{array}$ & $\begin{array}{l}6.4 \times 3.2 \times 3.2 \\
3.6 \times 6.4 \times 3.6\end{array}$ & $\begin{array}{l}64 \times 32 \times 32 \\
128 \times 256,18 \text { slices }\end{array}$ & $\begin{array}{l}4 \\
4\end{array}$ & $\begin{array}{l}2.7 \\
1.4\end{array}$ & $\begin{array}{l}32 \\
4\end{array}$ & 0.35 and 0.2 \\
\hline tomato, HT & ${ }^{23} \mathrm{Na}$ & small & 3D MSME & $3.2 \times 3.2 \times 3.2$ & $32 \times 32 \times 32$ & 4 & 10.9 & 128 & 0.07 \\
\hline tomato, $\mathrm{LT}$ & ${ }^{23} \mathrm{Na}$ & small & 3D MSME & $3.2 \times 3.2 \times 3.2$ & $32 \times 32 \times 32$ & 4 & 10.9 & 128 & 0.13 \\
\hline tomatoes, RSA & ${ }^{1} \mathrm{H}$ & small & 2D MSME & $3.2 \times 3.2 \times 3.2$ & $256 \times 256,64$ slices & 4 & 1.4 & 4 & \\
\hline
\end{tabular}

* Bruker's notation MSME means multi slice multi echo. For 3D only one slice was selected, therefore it is in fact a single slice multi echo sequence with $3 \mathrm{D}$ encoding. RARE is rapid acquisition with relaxation enhancement

\# number of averaged echoes

${ }^{+}$number of scans

$\S$ volumetric water content at time of scanning

\section{Results}

For an optimal setup of an imaging pulse sequence one should first investigate the relaxation times of the NMR active substance in the investigated system. Therefore we started this study with the investigation relaxation times of $\mathrm{NaCl}$ solutions in the given porous medium. Figure 2 shows the obtained relaxation spectra for ${ }^{23} \mathrm{Na}$ in FH31 sand and, for comparison, the corresponding spectra of ${ }^{1} \mathrm{H}$, taken from Stingaciu et al. (2010) and Stingaciu et al. (2009). Both ${ }^{23} \mathrm{Na}$ relaxation spectra were essentially mono-exponential with $T_{1, \mathrm{av}}=47 \mathrm{~ms}$, and $T_{2, \text { eff }}=43 \mathrm{~ms}$. It can be seen that the rapid transversal mode at about $5 \mathrm{~ms}$ contributed to less than $5 \%$ to the total relaxation process and is therefore negligible. Most

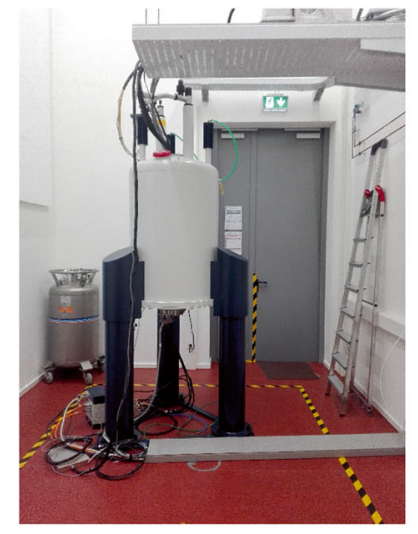

a)

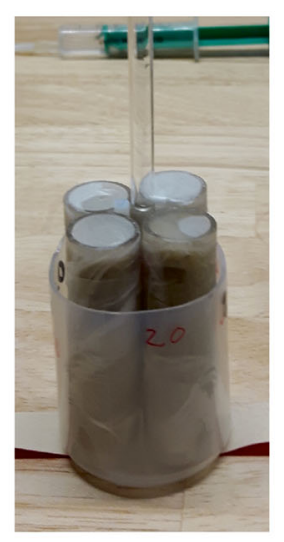

b)

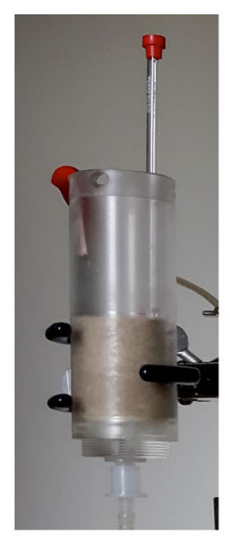

c)
Fig. 1 The experimental setup a) Bruker 4.7 T SWB MRI scanner, b) columns with four different $\mathrm{Na}^{+}$concentration for calibration, c) evaporation columns with liquid supply through a porous

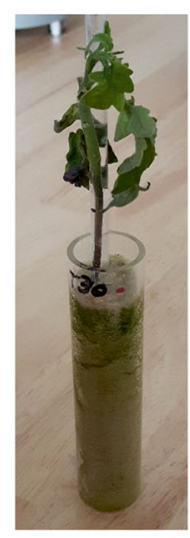

d)

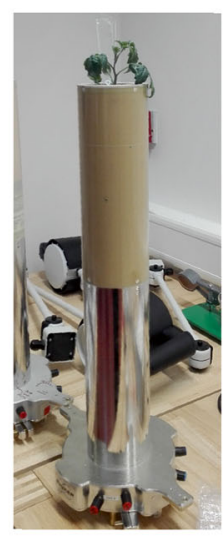

e) obvious is that the average values of $T_{1}$ and $T_{2, \mathrm{eff}}$ for ${ }^{23} \mathrm{Na}$ were almost identical. Furthermore, they also match the values found in pure aqueous solution (Rijners et al. 2004) and any dependence of $1 / T_{2, \text { eff }}$ on the echo time was not observed. Thus, a possible contribution of diffusion in internal gradients was negligible. In other words, the first terms in Eqs. 2a and b, i.e. bulk relaxation, dominate over the further contributions. It is in agreement with the findings of Rijners et al. (2004) and Kausik et al. (2018) who observed a significant acceleration of the transverse relaxation by the surface (i.e. the second term in Eq. 2) only for mesoporous systems with a pore size of less than $1 \mu \mathrm{m}$. The consequences for our investigations are i) that averaging of the first few echoes with an echo time of $2.6 \mathrm{~ms}$ will

plate at the bottom at a given hydraulic head and equipped with a vertical calibration tube inside, d) tomato plant growing in sandfilled glass column and e) tomato plant inside MRI coil 
have no influence on the proportionality between signal intensity and bulk $\mathrm{Na}$ concentration, and ii) that a relatively short repetition time of $t_{\mathrm{R}}=0.3 \mathrm{~s}$ can be set. It allows a high number of accumulations in a reasonable time even for 3D pulse sequences.

Calibration If the parameters $t_{\mathrm{R}}$ and $t_{\mathrm{E}}$ in Eq. 1 are set appropriately, i.e. $t_{\mathrm{R}}>5 \times T_{1}$ and $t_{\mathrm{E}}<<T_{2 \text {,eff }}$, the intensity in a voxel is proportional to the volume concentration of $\mathrm{Na}^{+}$in the unsaturated porous medium, $\mathrm{c}_{\mathrm{Na}}$, vol.

$c_{\mathrm{Na}, \mathrm{vol}}=n_{\mathrm{Na}} / V_{\mathrm{bulk}}=c_{\mathrm{Na}, \mathrm{sol}} \theta$.

In Eq. 3, $c_{\mathrm{Na} \text {,sol }}$ is the solution concentration, $n_{\mathrm{Na}}$ is the number of moles, $V_{\text {bulk }}$ is the bulk volume, and $\theta$ is the volumetric water content. Figure 3 summarizes the average intensities of the individual calibration tubes with different concentrations of $\mathrm{NaCl}$ (Fig. 1b) in a central axial slice at different degrees of saturation, plotted as a function of $c_{\mathrm{Na} \text {,sol }} \theta$ according to Eq. 3. The different degrees of saturation were achieved by water extraction, not by evaporation, so that the solution concentration $c_{\mathrm{Na}}$, sol in the porous medium remained constant while the volumetric concentration decreased. The linear relation proves the validity of the above assumptions that the terms in square brackets in Eq. 1 were close to unity for the given parameters. Thus, the MRI

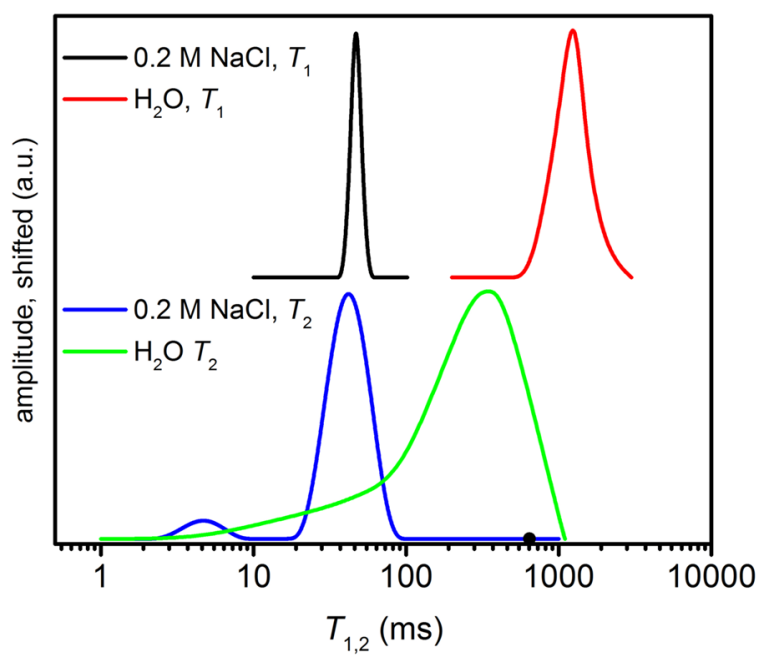

Fig. 2 Relaxation spectra for $0.2 \mathrm{M} \mathrm{NaCl}$ solution and pure water in FH31 sand obtained by inverse Laplace transformation of CPMG, and inversion recovery curves for $T_{2 \text {,eff }}$ and $T_{1}$, respectively. The data for pure water were taken from Stingaciu et al. (2009) signal was clearly proportional to the volume concentration.

Evaporation The second step was the investigation of $\mathrm{Na}^{+}$accumulation in pure soil by evaporation. Two scenarios under steady-state evaporation conditions were monitored: i) high, approximately constant water content related to a small hydraulic head of -10 at the bottom of the column and ii) lower water content combined with a distinct moisture profile related to a hydraulic head of $-20 \mathrm{~cm}$ at the bottom of the column. Fig. 4a depicts vertical water content profiles, which were obtained by normalizing the average, depth depending MRI signal to that of the marker tube. The water content for $h=-10 \mathrm{~cm}$ was relatively uniform; it decreased only slightly from $0.37 \mathrm{~cm}^{3} / \mathrm{cm}^{3}$ at the bottom to $0.34 \mathrm{~cm}^{3} / \mathrm{cm}^{3}$ at the top. Lowering of the hydraulic head to $h=-20 \mathrm{~cm}$ resulted in a more pronounced profile between $0.37 \mathrm{~cm}^{3} / \mathrm{cm}^{3}$ at the bottom to $0.1 \mathrm{~cm}^{3} / \mathrm{cm}^{3}$ at a depth of $-0.2 \mathrm{~cm}$. A shallow dry layer developed on the surface which caused a significant reduction of the evaporation rate due to loss of capillary continuity (Merz et al. 2016; Shokri et al. 2010). These different scenarios resulted in different evaporation rates

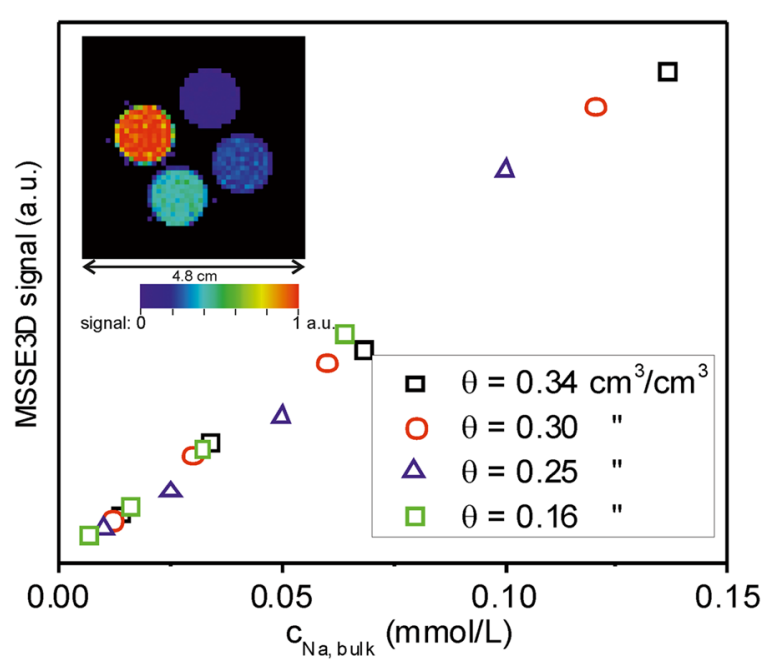

Fig. 3 Result of the calibration experiment, Fig. 1b, scanned at different water contents using a 3D-multislice multi echo sequence (3D MSME). The insert depicts an axial cross section through the column showing the four cuvettes with different solution concentrations of $\mathrm{NaCl}$ at $\theta=0.34 \mathrm{~cm}^{3} / \mathrm{cm}^{3}$. On the ordinate the average MRI signals of the individual cuvettes with solution concentrations of $c_{\mathrm{Na} \text {, sol }}=0.04,0.10,0.20$, and $0.40 \mathrm{~mol} / \mathrm{L}$ are plotted. By water extraction the different degrees of saturation are adjusted, yielding the different volume concentrations according to $c_{\mathrm{Na}, \text { bulk }}=n_{\mathrm{Na}} / V_{\text {bulk }}=c_{\mathrm{Na}} \theta$, plotted on the abscissa 
of $1.0 \mathrm{~cm} /$ day and $0.51 \mathrm{~cm} /$ day for the high and low water content scenarios, respectively (Fig. 4b). It led to distinct differences in development of the $\mathrm{Na}^{+}$distributions shown in Fig. 5a and c. Zones with locally stronger accumulation were observed near the top and, for the high water content scenario on day 7 , above a local depletion zone (Fig. 5a, right panel, yellow arrow). A possible explanation for this phenomenon is lower local flow velocity promoting the local accumulation.

The distribution patterns are best described by the vertical concentration profiles, obtained by averaging the $\mathrm{Na}^{+}$concentration in each depth (Fig. 5b and d). With the high water content conditions coupled to a higher evaporation rate, $\mathrm{Na}^{+}$accumulation was higher than in the low water content scenario. In both cases, a shallow surface layer of high concentration developed after one day. In contrast, the $\mathrm{Na}^{+}$distribution remained homogeneous at depths below $-0.3 \mathrm{~cm}$ and reflected the vertical water content distribution after one week of evaporation. This was especially true for the low water content scenario, where the vertical Na-profile (Fig. 5c) matched the vertical water content profile (Fig. 4a, blue curve). Even the edge at $-3 \mathrm{~cm}$ depth was mirrored. We conclude that dispersive spreading exceeds the advective accumulation of $\mathrm{Na}^{+}$at these given pore flow velocities.

The total amount of $\mathrm{NaCl}$ inside the column was calculated from the average $\mathrm{Na}^{+}$concentration in the entire soil column by multiplication with the volume of the packing. The comparison of this MRI-derived amount with the total inflow amount, obtained from the total evaporation rate times the input solution concentration is shown in Fig. 6. The data agreed well for both scenarios. This closure of the mass balance proves that the given approach, i.e. the used MRI pulse sequence and its parameters and the data evaluation by normalization on the marker tube, yielded reliable $\mathrm{Na}^{+}$concentration maps even in the low concentration range above $0.01 \mathrm{~mol} / \mathrm{L}$. Such low concentrations of $\mathrm{Na}^{+}$could be imaged quantitatively with the chosen spin-echo sequence, analogous to applications on plants (Rokitta et al. 2004) and in medicine and biology using gradient echo sequences (Kopp et al. 2012). Complications by relaxation acceleration occurring at high concentrations were not encountered, which would require the application of MRI methods with very rapid detection after excitation such as SPRITE (Veliyulin et al. 2009). However, such methods should be taken into consideration if the relaxation of ${ }^{23} \mathrm{Na}$ is significantly accelerated e.g. in clayey and loamy soils.

Accumulation in tomato root systems After we showed that MRI can quantitatively monitor the $\mathrm{Na}^{+}$accumulation in soil, we applied the method in the last step for imaging of $\mathrm{Na}^{+}$accumulation in the root zone of tomato plant systems. The plants were grown from seeds for six weeks under two transpiration regimes. They were regularly irrigated with saline water corresponding to an electrical conductivity (EC) of $4.5 \mathrm{dSm}^{-1}$ $(\sim 0.042 \mathrm{~mol} / \mathrm{L})$. The average transpiration rate for the high transpiration regime (HT) under $60 \%$ relative humidity conditions was $1.2 \mathrm{~cm}^{3} /$ day $(0.54 \mathrm{~cm} /$ day $)$, whereas it reduced to $0.68 \mathrm{~cm}^{3} /$ day $(0.30 \mathrm{~cm} /$ day $)$ in the low transpiration regime under nearly $100 \%$ relative humidity (LT). After 6 weeks of development distinct differences in the complexity of the RSA became obvious. The RSA of tomato in the HT regime (Fig. 7a) was considerably more complex than that in the LT regime (Fig. 7e). Also, there was more $\mathrm{Na}^{+}$accumulation in the top soil region and in the taproot, visible in the slice at $+0.8 \mathrm{~cm}$ above the center of field-of-view $(\mathrm{FoV})$ of the $\mathrm{Na}^{+}$sensitive images, see Fig. 7c. This becomes clearer when inspecting axial slices, three of which are shown in Fig. $7 b$ and $d$ for the RSA and the $\mathrm{Na}^{+}$distribution, respectively. Strong accumulation was found $0.8 \mathrm{~cm}$ above the center of FoV, but also in deeper layers (at the center of FoV and at $-0.8 \mathrm{~cm}$ below FoV). The accumulation around roots was less pronounced for the low transpiration scenario, Fig. $7 \mathrm{~g}$ and h. For example, under LT, roots are barely visible in the $\mathrm{Na}^{+}$scan, while under HT the shape of the roots is very clear, meaning higher $\mathrm{Na}^{+}$accumulation at the roots or even inside. The resolution of $1 \mathrm{~mm}$ for the $\mathrm{Na}^{+}$sensitive images did not allow differentiation between accumulation at the soil-root surface and uptake, see the discussion in the next section. The next step was the quantitative analysis of the $\mathrm{Na}^{+}$distribution in the part of the column indicated by the dotted arrows in Figs. $7 \mathrm{c}$ and g. The vertical concentration profiles in Fig. 8 show a higher accumulation in the HT compared to the LT scenario. In the center and bottom region, the concentrations were comparable, but in the topmost $1 \mathrm{~cm}$ it was about a factor two higher in the HT scenario.

The calibrated images of $\mathrm{Na}^{+}$scans allowed the calculation of $\mathrm{Na}^{+}$concentrations as a function of distance from the root, exemplarily shown in in Fig. 9. A decrease in $\mathrm{Na}^{+}$concentration was observed even in a 
a)

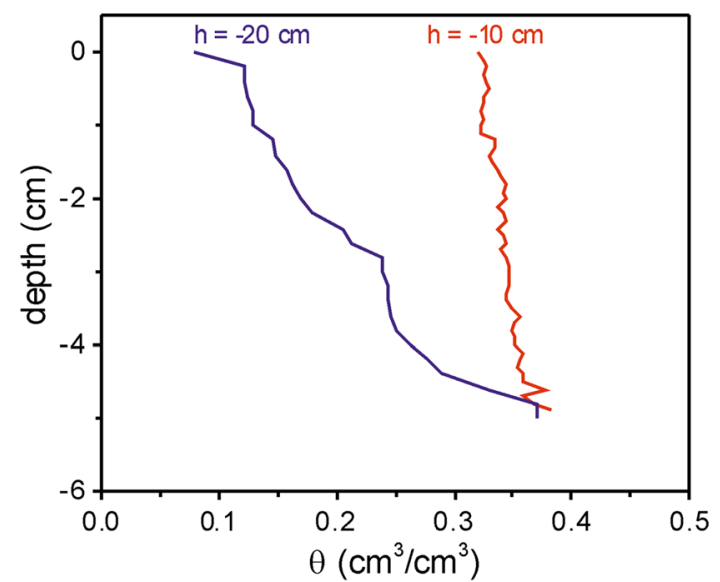

b)

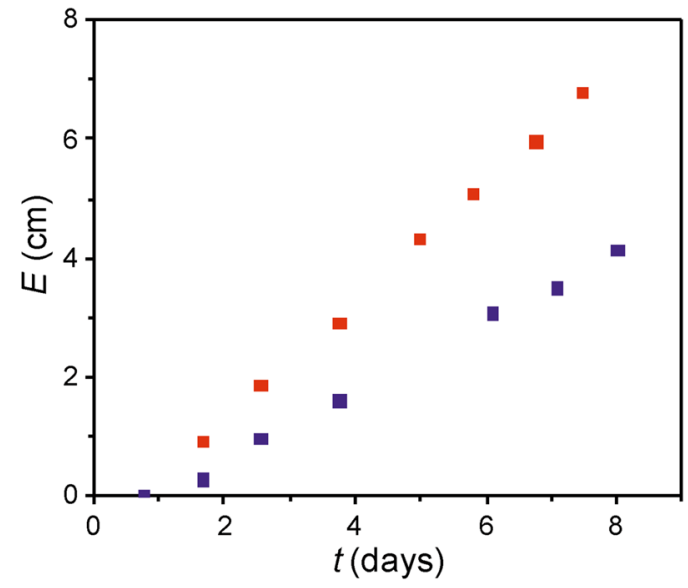

Fig. 4 a Water content vs. depth for the evaporation scenarios at different hydraulic heads of $-10 \mathrm{~cm}$ and $-20 \mathrm{~cm}$ at the infiltration plate at the bottom of the column. Zero refers to the soil surface. b Total evaporation $(E)$ with time

short distance as $2 \mathrm{~mm}$ away from the root interface, where the decrease in $\mathrm{Na}^{+}$concentrations from the root interface appeared steeper under HT than under LT conditions.
The construction of distance - concentration correlation plots allowed the calculation of this behavior for the entire root system in the region of $+-1.5 \mathrm{~cm}$ above and below the center of FoV of the Na-sensitive images. The a)

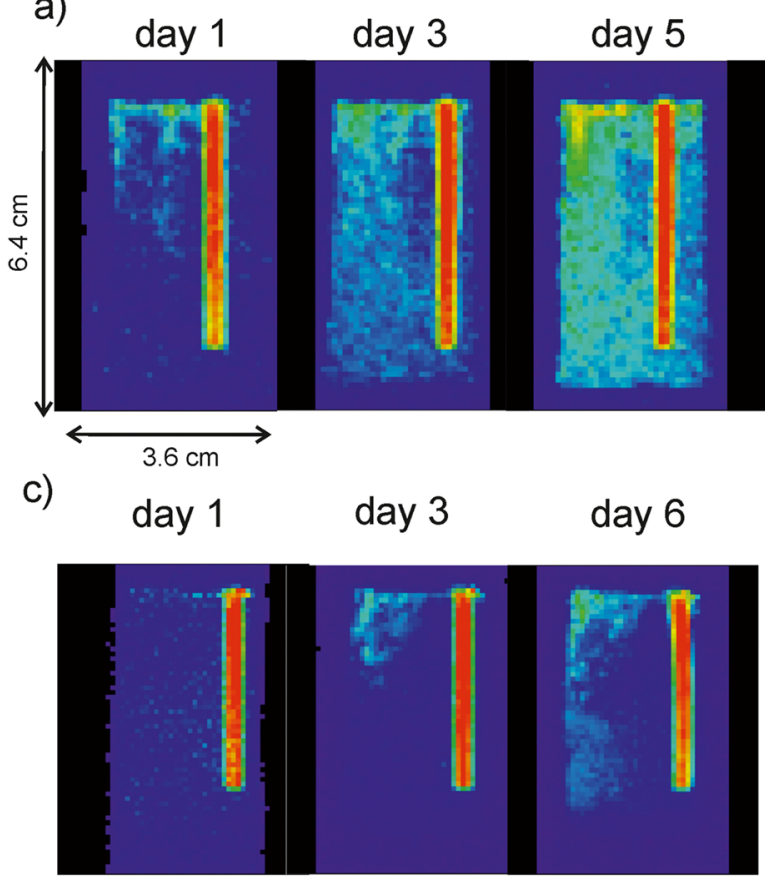

Fig. 5 Results of the evaporation experiment.. Top row: The series monitored at $\mathrm{h}=-10 \mathrm{~cm}$ corresponding to high water content. Bottom row: $\mathrm{h}=-20 \mathrm{~cm}$ corresponding to low water content. a and $\mathbf{c}$ Maximum intensity projections of the $\mathrm{Na}^{+}$distribution at b)
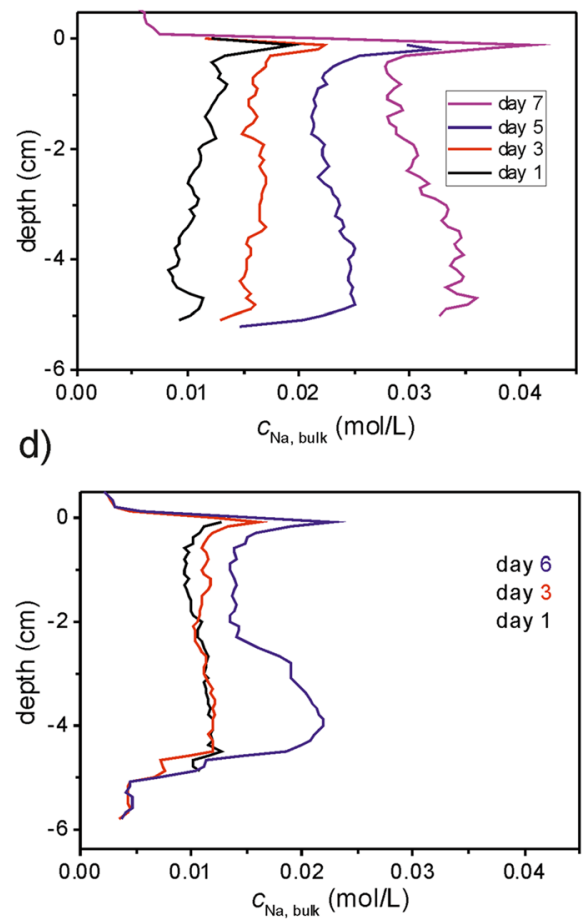

selected dates. The resolution is $1 \mathrm{~mm}$ isotropic. The yellow arrow points to a zone with locally higher $\mathrm{Na}^{+}$concentrations. $\mathbf{b}$ and $\mathbf{d}$ Bulk $\mathrm{Na}^{+}$concentration profiles on different days after starting 


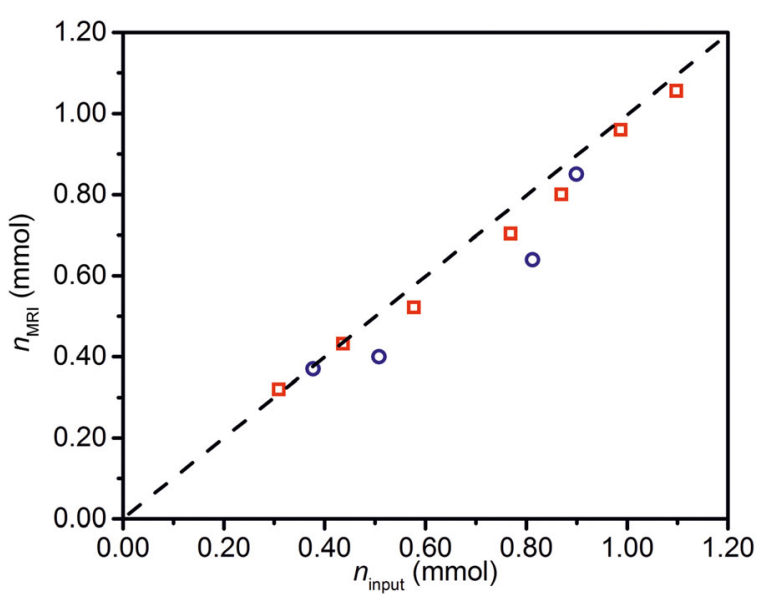

Fig. 6 Comparison of input amount, calculated from the transpired volume times the original concentration, and the amount of $\mathrm{Na}$ recovered by integration of the ${ }^{23} \mathrm{Na}-\mathrm{MRI}$ images over the entire cuvette and normalization on the calibration tube. $\square$ : High water content at $h=-10 \mathrm{~cm}, \mathrm{O}$ : Low water content at $h=-20 \mathrm{~cm}$

first step was the calculation of the distance transform map of the binary RSA images (see the example in Fig. 10a). It yielded multislice distance maps of which one slice is shown as an example in Fig. 10b). Next, the distance - concentration profiles correlation diagrams were constructed by plotting the Na-concentration vs. the distance to the nearest root for each voxel. The result is shown in Figs. 10d and e for the HT and LT scenarios, respectively. It showed a stronger accumulation of $\mathrm{Na}^{+}$ near the roots under HT conditions, since the maximum is more pronounced, and the decay is shorter. The noise in the concentration-distance profiles agreed with observations from other tracer experiments in soil-root systems (Haber-Pohlmeier et al. 2017). It could be explained using model simulations by the impact of several roots on the accumulation of a tracer/salt at a certain location, by the fact that water uptake and accumulation varies between different root segments, and by the impact of 3D transport (Koch et al. 2019).

\section{Discussion}

The results from the calibration experiment showed that MRI could detect $\mathrm{Na}^{+}$volume concentrations in soil also under unsaturated conditions. The relaxation times of ${ }^{23} \mathrm{Na}^{+}$in the sand matrix were identical to the bulk relaxation times, and no influence of the porous medium by surface enhanced relaxation or internal gradients was observed. This can be explained by the relatively high bulk relaxation rate which dominates over the other terms in Eq. 2. Moreover, the concentration range is sufficiently low that changes in the hydration state of ${ }^{23} \mathrm{Na}^{+}$plays no role. This is in agreement with the findings of Rijners et al. (2004). The facts that i) the relaxation in medium-sized porous media was controlled by bulk relaxation and that ii) a second, more rapid relaxing mode was not detected, is advantageous for $\mathrm{Na}^{+}$imaging. It allowed to set up a spin density controlled pulse sequence by using a moderately short echo time of $3.4 \mathrm{~ms}$ and a sufficiently long repetition time of greater than 5 times $T_{1}$. A significant impact of the porous medium is only expectable for significantly smaller pore sizes of e.g. silt material. The approach was validated by the calibration experiment where we found that the image intensity is proportional to the volume concentration of $\mathrm{Na}^{+}$down to water content of $0.15 \mathrm{~cm}^{3} /$ $\mathrm{cm}^{3}$. Furthermore we could demonstrate by both evaporation experiments that this type of $\mathrm{Na}^{+}$imaging yields quantitative sodium concentration maps and the mass balance was closed. The recovery rates were close to $100 \%$. The evaporation experiments tell us further that $\mathrm{NaCl}$ is highly mobile in the system and we conclude that the diffusional redistribution compensates possible advective enrichment toward the top (Nachshon et al. 2011; Nachshon et al. 2018; Shokri et al. 2010).

RSA scans were performed at relatively low water contents to obtain good contrast between the roots and soil. The plants were grown in the tubes from seeds for six weeks, a period, which is long enough to detect differences in root development between HT and LT, but not too long to create an over-crowded root system. Please note that the root systems of such young plants were not far developed. They can be imaged well by MRI as shown by Metzner et al. (2015) and HaberPohlmeier et al. (2019). In the latter paper, we compared also the detectability of the edges and diameters of cylindrical structures in wet sand, mimicking roots, by $\mathrm{X}$-ray-CT, neutron-CT and MRI. Therefore, MRI imaging of roots is adequate for the assessment of the range of $\mathrm{Na}^{+}$accumulation since the given resolution of $1 \mathrm{~mm}$ for the ${ }^{23} \mathrm{Na}$ images is coarser than the diameter of possible fine roots. We therefore refrained from excavating the roots and further analyzing the root system architecture.

Sodium MRI scans were performed on the same day as the RSA scans, thus it is important to assure that the visibility of $\mathrm{Na}^{+}$is not compromised due to the low 


\section{high transpiration}

a)

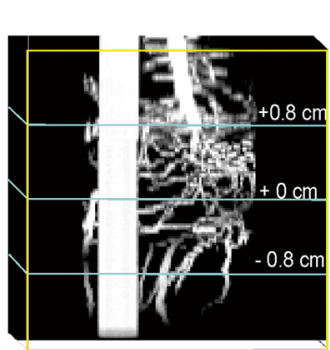

c)

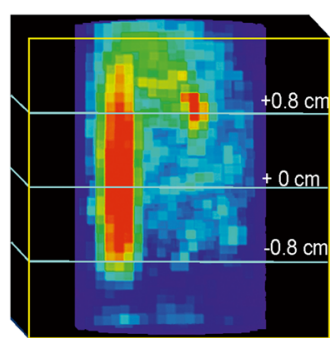

b)

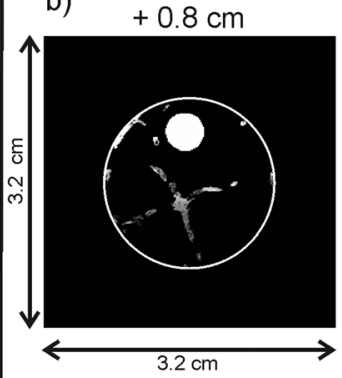

d)

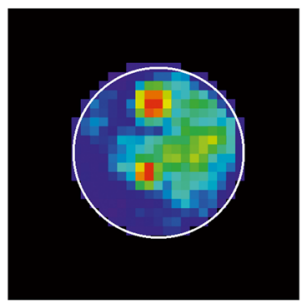

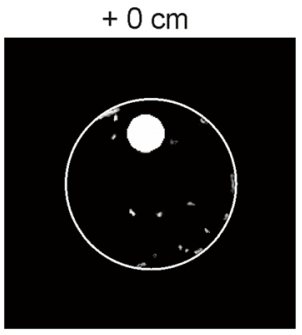
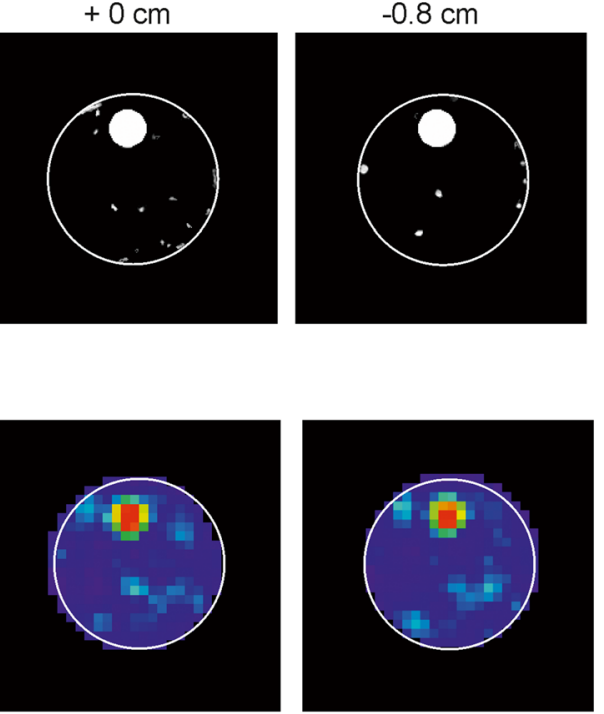

$\mathrm{C}_{\mathrm{Na}}: 0, \quad, \quad+50 \mathrm{mM}$ low transpiration

e)

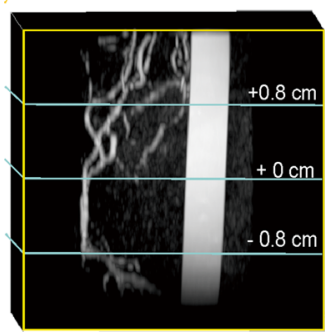

g)

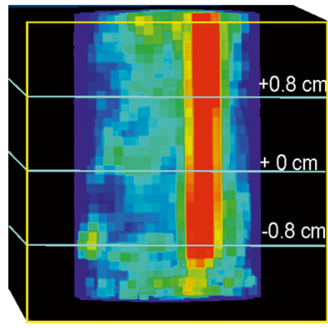

f)

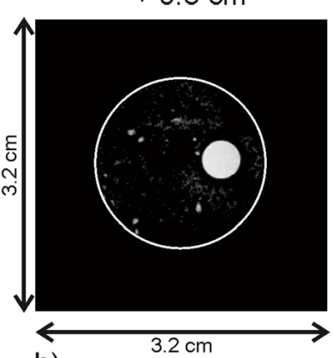

h)

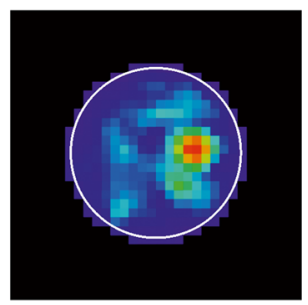

$+0 \mathrm{~cm}$
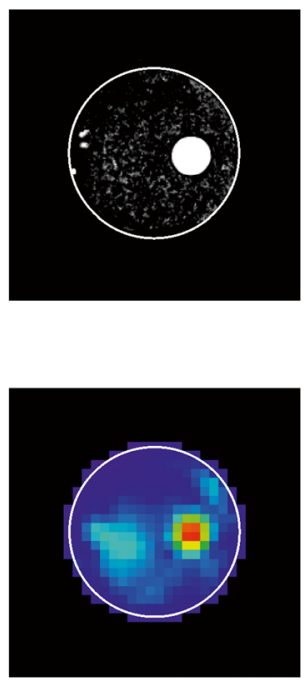

$\mathrm{C}_{\mathrm{N}:}: 0$

c. $50 \mathrm{mM}$
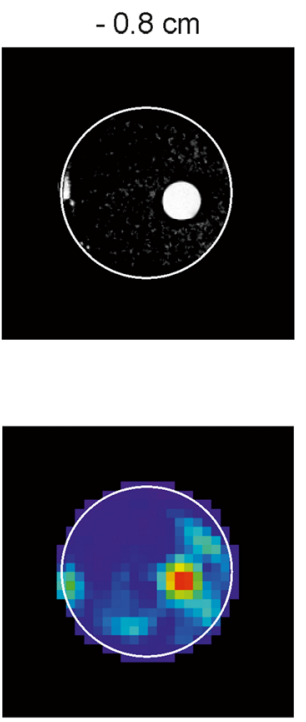

indicate the selected axial cross sections at $+0.8 \mathrm{~cm}, 0 \mathrm{~cm}$, and $0.8 \mathrm{~cm}$ with respect to the center of the field of view. The right panels are selected axial cross sections for the root sensitive images, $\mathbf{b}$ and $\mathbf{f}$, and the $\mathrm{Na}^{+}$images, $\mathbf{d}$ and $\mathbf{h}$. The bright circles are the marker tube, filled with $0.05 \mathrm{~mol} / \mathrm{L} \mathrm{NaCl}$ solution

with the calibration experiment shows proportionality between the ${ }^{23} \mathrm{Na}$ MRI signal and the volume

Concerning the detectability of $\mathrm{Na}^{+}$, the comparison 


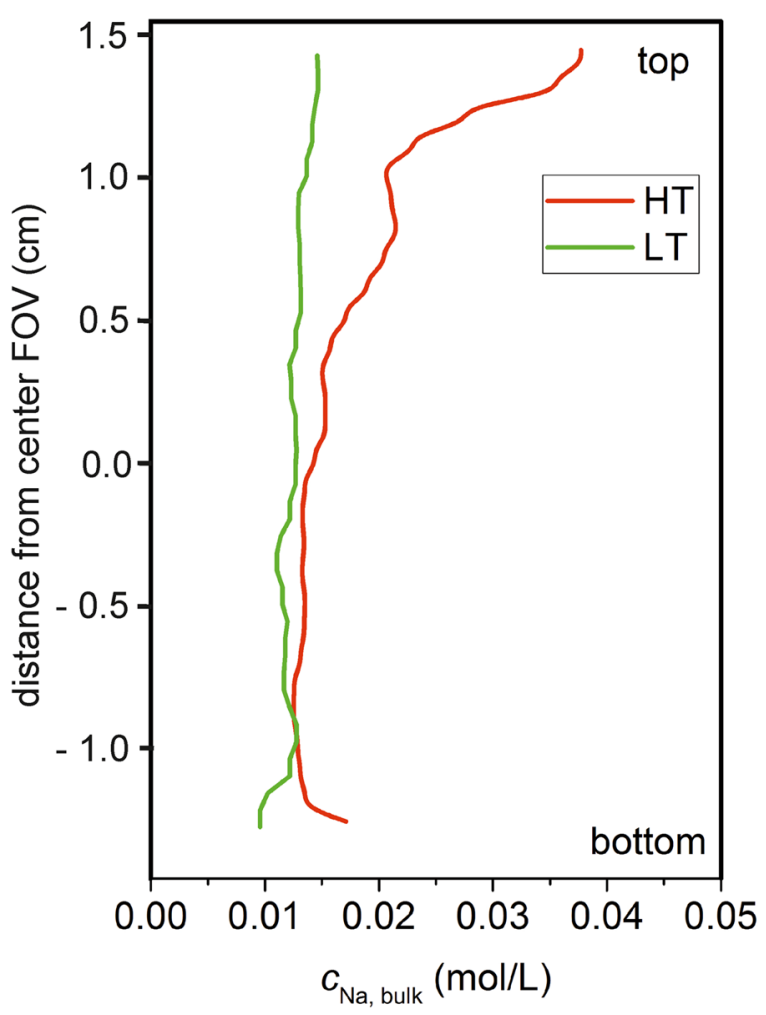

Fig. 8 Average bulk Na-concentrations as a function of depth in the range, indicated by the dotted arrows in Fig. 7 c) and g). The data are obtained by integration over each individual cross section excluding the area of the marker tube. Three cross sections are exemplarily shown in Fig. $7 \mathrm{~d}$ ) and h). HT and LT refer to the high and low transpiration scenarios

concentration at comparable water contents and no interference is expected. This is also supported by the 2 nd evaporation experiment, where the recovery rate was close to $100 \%$ although the water content near the surface was below $0.1 \mathrm{~cm}^{3} / \mathrm{cm}^{3}$.

The analysis of the $\mathrm{Na}^{+}$distribution around single roots under low and high transpiration rates showed different accumulation patterns. Less $\mathrm{Na}^{+}$accumulated around the roots under LT conditions and its distribution was more homogenous through the soil profile. Under $\mathrm{HT}$, higher concentrations of $\mathrm{Na}^{+}$were found around the roots and more in the upper soil layers in comparison to LT conditions, probably due to higher transpiration. The entire column was not scanned since it would require three different scans at three depths, which would have been time consuming. The duration of a single scans is included in Table 1.

These results support evidence found in former studies that $\mathrm{Na}^{+}$accumulation was generally increased in the root zone or at the interface of a single root under high
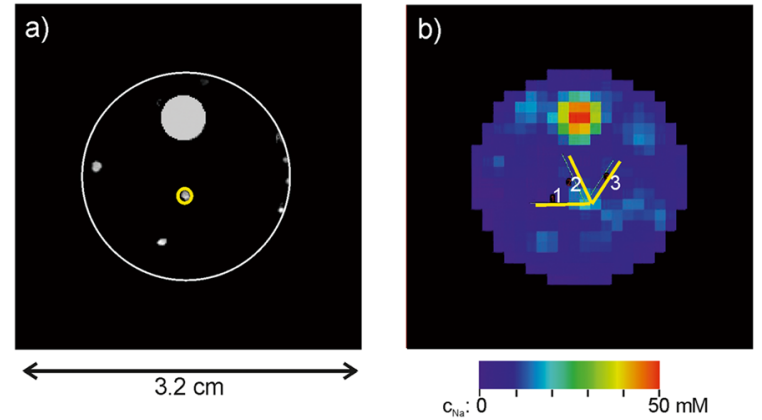

c)
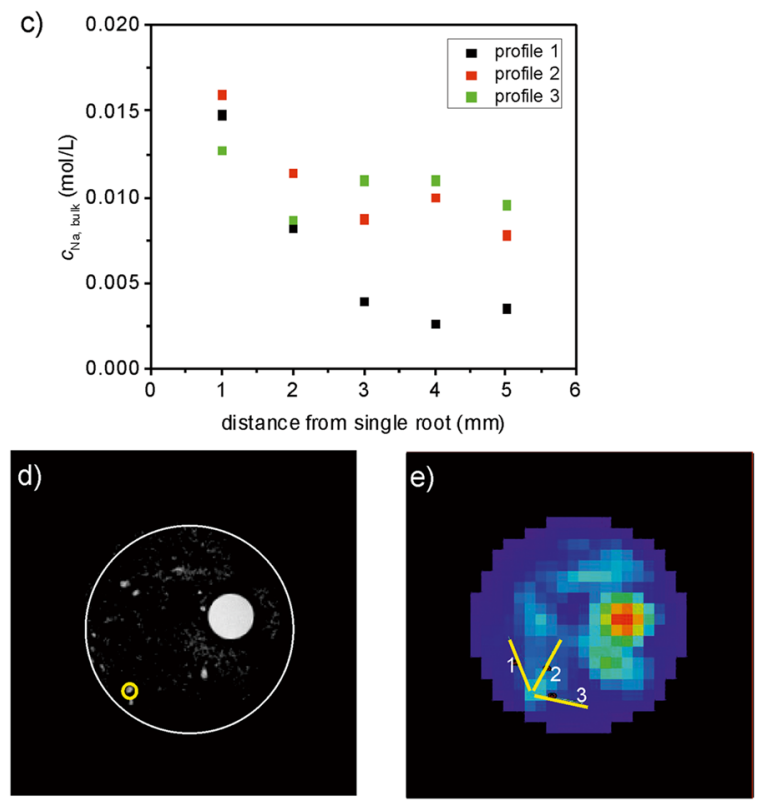

f)

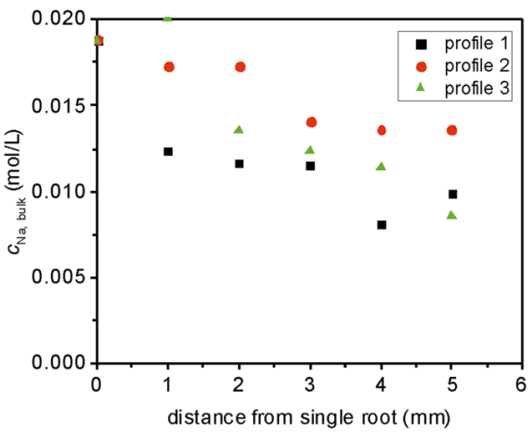

Fig. 9 Construction of axial Na concentration profiles for LT (top half) and HT (bottom half) plants. a and $\mathbf{d}$ are axial slices of the RSA images, $\mathbf{b}$ and $\mathbf{e}$ are the corresponding bulk Na concentration maps. For a given selected lateral root, axial profiles are calculated in three directions, indicated by the numbers 1 to 3; these are depicted in $\mathbf{c}$ for the LT and f) for the HT scenario

transpiration rates (Hamza and Aylmore 1992; Jorda et al. 2018; Perelman et al. 2020; Riley and Barber 1970; Sinha and Singh 1974). Nevertheless, the present 
a)

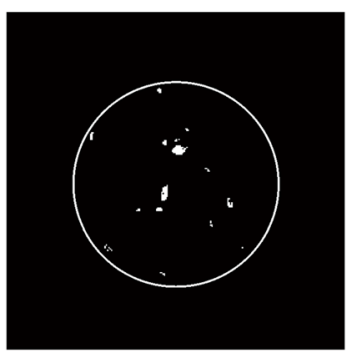

b)

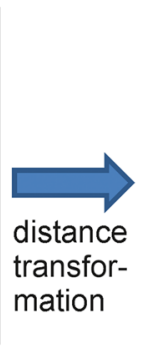

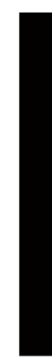
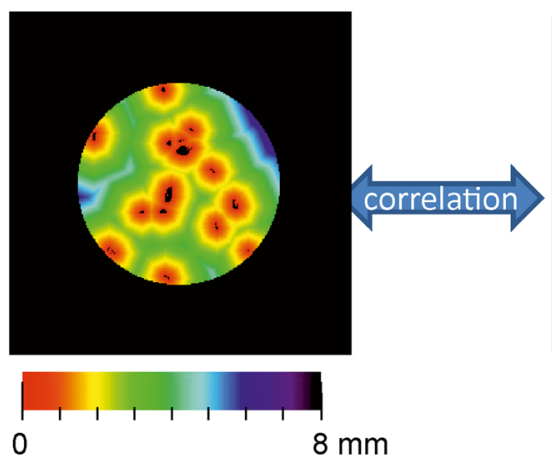

c)

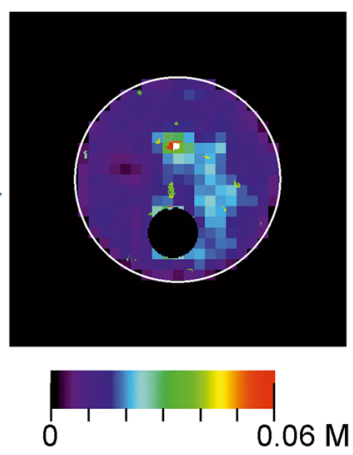

d)

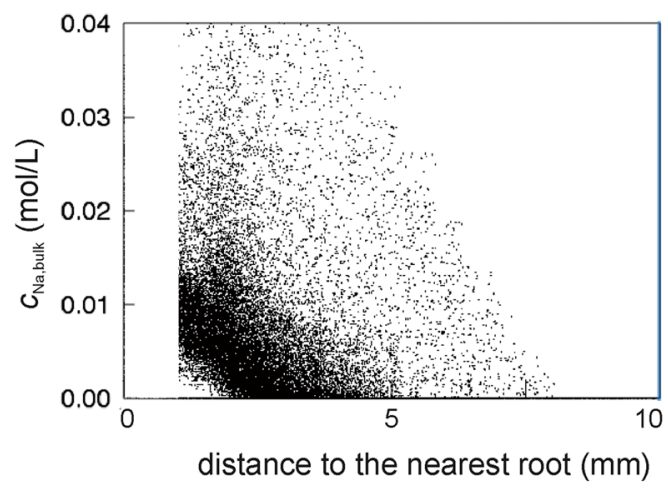

Fig. 10 Construction of concentration-distance correlation diagrams for the HT (d) and LT (e) scenarios. a First the roots are segmented and binarized individually for each axial slice. b By using a Euklidean distance transformation one obtains for each

MRI study provides finer resolution of down to $1 \mathrm{~mm}$ and thus enables measuring $\mathrm{Na}^{+}$gradients from the root interface in a range of few millimeter. The sample size is limited by the inner diameter of the rf-coil. For our system it is $66 \mathrm{~mm}$ diameter and a vertical field of view of $70 \mathrm{~mm}$. The sample size should also not be significantly smaller than the rf coil, since the sensitivity scales with the ratio of the sample volume and the void volume of the coil. Therefore, we have used for most of our studies a smaller rf-coil and gradient system, which was inserted into the built-in system of the Bruker SWB magnet.

The low gyromagnetic ratio $\gamma$ of ${ }^{23} \mathrm{Na}$ combined with low volume concentrations $(0.02 \mathrm{mmol} / \mathrm{L}$ compared to ca. $16 \mathrm{~mol} / \mathrm{L}$ for water saturated sand) requires the usage of 3D MRI pulse sequences instead of multi-slice sequences, which are significantly faster. Also, the number of scans are significantly higher. The consequence are long scanning times - up to $12 \mathrm{~h}$, and relatively low resolution of the image (compared to the ${ }^{1} \mathrm{H}^{+}$scan).

\section{e)}

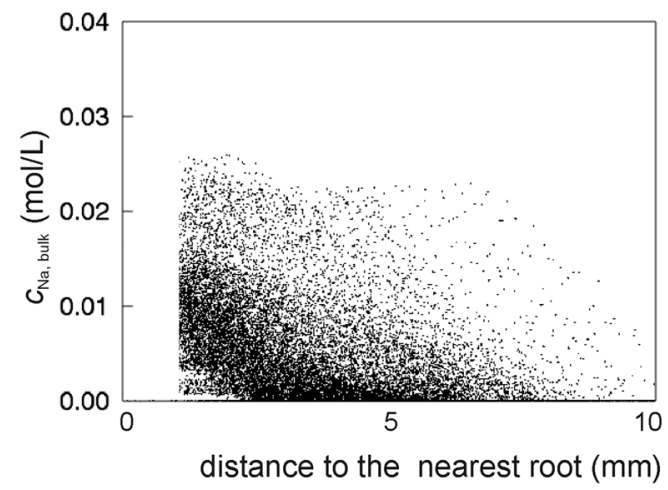

slice a map with rainbow-encoded distance of each voxel to the nearest root. The respective Na concentrations for each voxel are finally plotted vs. its distance, resulting in the correlation diagrams in d) and e)

Long scanning times also led to low number of repetitions, which will require further research. This issue might be overcome in future by the use of MRI pulse sequences with ultrafast detection times such as SPRITE or UTE due to the significantly shorter repetition time (Konstandin et al. 2015; Romanzetti et al. 2006). A second advantage of these type of sequences is the higher resolution of up to 128 pixel per dimension compared to 32 to 48 . This will allow also the investigation of longer samples by matching different scans.

Future studies combing data from MRI scans and root uptake models can help to induct processes on a field scale. Despite its limitations, MRI can provide a finer resolution of $\mathrm{Na}^{+}$accumulation on the root-soil interface and improve existing knowledge about processes and interaction between the root and the soil.

Acknowledgements This research was supported by the Grant I-62-204.4-2013 from the German-Israeli Foundation for Scientific Research and Development and by the Israel Ministry of 
Agriculture and Rural Development (Eugene Kandel Knowledge Centers) as part of the "Root of the Matter: The root zone knowledge center for leveraging modern agriculture."

Funding Information Open Access funding provided by Projekt DEAL.

Open Access This article is licensed under a Creative Commons Attribution 4.0 International License, which permits use, sharing, adaptation, distribution and reproduction in any medium or format, as long as you give appropriate credit to the original author(s) and the source, provide a link to the Creative Commons licence, and indicate if changes were made. The images or other third party material in this article are included in the article's Creative Commons licence, unless indicated otherwise in a credit line to the material. If material is not included in the article's Creative Commons licence and your intended use is not permitted by statutory regulation or exceeds the permitted use, you will need to obtain permission directly from the copyright holder. To view a copy of this licence, visit http://creativecommons.org/licenses/by/4.0/.

\section{References}

Barber SA (1962) A diffusion and mass-flow concept of soil nutrient availability. Soil Sci 93:39-49

Billich C, Muche R, Brenner G, Schmidt SA, Krüger S, Brambs HJ, Pauls S (2008) CT-guided lung biopsy: incidence of pneumothorax after instillation of $\mathrm{NaCl}$ into the biopsy track. Eur Radiol :1146-1152

Blümich B (2000) NMR imaging of materials. Clarendon Press, Oxford

Blümich B, Haber-Pohlmeier S, Zia W (2014) Compact NMR. De Gruyter, Berlin

Callaghan PT (1991) Principles of nuclear magnetic resonance microscopy. Oxford University Press, Oxford

Coates GR, Xiao LP, Prammer M.G. (1999) NMR logging principles and applications vol http://www.nfes.org/archive/2009 /SeminarPDF/01_Book.pdf. HalliburtonEnergyServices, Houston

Denmead OT, Shaw RH (1962) Availability of soil water to plants as affected by soil moisture content and meteorological conditions. Agron J 54:385-390

Flavel RJ, Guppy CN, Tighe M, Watt M, McNeill A, Young IM (2012) Non-destructive quantification of cereal roots in soil using high-resolution X-ray tomography. J Exp Bot 25032511:2503-2511. https://doi.org/10.1093/jxb/err421

Haber-Pohlmeier S, Vanderborght J, Pohlmeier A (2017) Quantitative mapping of solute accumulation in a soil-root system by magnetic resonance imaging. Water Resour Res 53:7469-7480. https://doi.org/10.1002/2017WR020832

Haber-Pohlmeier S, Tötzke C, Oswald S, Blümich B, Pohlmeier A (2018) Imaging of root zone processes using MRI T1 mapping. Microp Mesop Mat:43-46 doi: https://doi.org/10.1016 j.micromeso.2017.10.046

Haber-Pohlmeier S, Tötzke C, Lehmann E, Kardjilov N, Pohlmeier A, Oswald S (2019) Combination of magnetic resonance imaging and neutron computed tomography for three-dimensional Rhizosphere imaging. Vadose Zone J 18: 180166-180111. https://doi.org/10.2136/vzj2018.09.0166

Hadrich JC (2011) Managing the economics of soil salinity North Dakota State University

Hamza MA, Aylmore LAG (1992) Soil Solute Concentration and Water-Uptake by Single Lupin and Radish Plant-Roots. I Water Extract Solute Accumulat Plant Soil 145:187-196

Hund A, Trachsel S, Stamp P (2009) Growth of axile and lateral roots of maize: I development of a phenotying platform. Plant Soil 325:335-349

Jorda H, Perelman A, Lazarovitch N, Vanderborght J (2018) Exploring osmotic stress and differences between soil-root Interface and bulk salinities. Vadose Zone J 17:170029. https://doi.org/10.2136/vzj2017.01.0029

Kausik R, Fellah K, Yang DHM (2018) Sodium NMR relaxation in mesoporous systems. Microporous Mesoporous Mater 269:134-137. https://doi.org/10.1016/j. micromeso.2017.03.009

Koch A, Meunier F, Vanderborght J, Garre S, Pohlmeier A, Javaux M (2019) Functional-structural root-system model validation using a soil MRI experiment. J Env Bot 70: 2797-2809. https://doi.org/10.1093/jxb/erz060

Koebernick N, Weller U, Huber K, Schlüter S, Vogel HJ, Jahn R, Vereecken H, Vetterlein D (2014) In Situ Visualization and Quantification of Three-Dimensional Root System Architecture and Growth Using X-Ray Computed Tomography. Vadose Zone J 13. https://doi.org/10.2136 /vzj2014.03.0024

Konstandin S, Kramer P, Gunther M, Schad LR (2015) Sodium magnetic resonance imaging using ultra-short echo time sequences with anisotropic resolution and uniform $\mathrm{k}$-space sampling. Magn Reson Imaging 33:319-327. https://doi. org/10.1016/j.mri.2014.12.007

Kopp C et al (2012) Na-23 magnetic resonance imaging of tissue sodium hypertension. Hypertension 59:167-172. https://doi. org/10.1161/hypertensionaha.111.183517

Kuchenbuch RO, Ingram KT (2002) Image analysis for nondestructive and non-invasive quantification of root growth and soil water content in rhizotrons. J Plant Nutr Soil Sci 165: $573-581$

Le Marié C, Kirchgessner N, Marschall D, Walter A, Hund A (2014) Rhizoslides: paper-based growth system for non-destructive, high throughput phenotyping of root development by means of image analysis. Plant Methods 10:13

Mairhofer S, Sturrock C, Wells DM, Bennett MJ, Mooney SJ, Pridmore TP (2015) On the evaluation of methods for the recovery of plant root systems from X-ray computed tomography images. Funct Plant Biol 42:460-470. https://doi. org/10.1071/fp 14071

McRobbie DW, Moore EA, Graves MJ, Prince MR (2007) MRI from picture to proton. Cambridge University Press, Cambridge

Merz S, Pohlmeier A, Balcom BJ, EnJilela R, Vereecken H (2016) Drying of a natural soil under evaporative conditions: a comparison of different magnetic resonance methods. App1 Magn Reson 47:212-138. https://doi.org/10.1007/s00723015-0736-6

Metzner R, Eggert A, van Dusschoten D, Pflugfelder D, Gerth S, Schurr U, Uhlmann N, Jahnke S (2015) Direct comparison of MRI and X-ray CT technologies for 3D imaging of root systems in soil: potential and challanges for root trait 
quantification. Plant Methods 11:17. https://doi.org/10.1186 /s13007-015-0060-Z

Mitchell J, Fordham EJ (2017) Sodium-23 NMR in porous media. Microp Mesop Mat

Nachshon U, Shahraeeni E, Or D, Dragila M, Weisbrod N (2011) Infrared thermography of evaporative fluxes and dynamics of salt deposition on heterogeneous porous surfaces. Water Resour Res 47. https://doi.org/10.1029/2011wr010776

Nachshon U, Weisbrod N, Katzir R, Nasser A (2018) NaCl crust architecture and its impact on evaporation: three-dimensional insights. Geophys Res Lett 45:6100-6108. https://doi. org/10.1029/2018gl078363

Neu TR, Manz B, Volke F, Dynes JJ, Hitchcock AP, Lawrence JR (2010) Advanced imaging techniques for assessment of structure, composition and function in biofilm systems. FEMS Microbiol Ecol 72:1-21. https://doi.org/10.1111 /j.1574-6941.2010.00837.x

Olt S, Krötz E, Komor E, Rokitta M, Haase A (2000) 23Na and $1 \mathrm{H}$ NMR microimaging of intact plants. J Magn Reson 144:297304. https://doi.org/10.1006/jmre.2000.2076

Pel L, Donkers PAJ, Kopinga K, Noijen JJ (2016) 1H, 23Na, and $35 \mathrm{Cl}$ imaging on Cementitious materials. Appl Magn Reson 47:265-276

Perelman A, Jorda H, Vanderborght J, Lazarovitch N (2020) Tracing root-felt sodium concentrations under different transpiration rates and salinity levels. Plant Soil 447:55-71. https://doi.org/10.1007/s11104-019-03959-5

Polomsky J, Kuhn N (2002) Root research methods. In: Beeckman T (ed) Plant roots: the hidden half. CRC Press, Boca Raton, pp 447-487

Rijners LA, Magusin PCMM, Huninik HP, Pel L, Kopinga K (2004) Sodium NMR relaxation in porous materials. J Magn Reson 167:25-30

Riley D, Barber SA (1970) Salt accumulation at the soybean (glycine max. (L.) merr.) root-soil interface. Soil Sci Soc Am J 34:154-155

Rodriguez P, Dell'amico J, Morales D, Sánchez Blanco MJ, Alarcón JJ (1997) Effects of salinity on growth, shoot water relations and root hydraulic conductivity in tomato plants. $\mathrm{J}$ Agric Sci 128:439-444

Rokitta M, Medek D, Pope JM, Critchley C (2004) Na-23 NMR microimaging: a tool for non-invasive monitoring of sodium distribution in living plants. Funct Plant Biol 31:879-887. https://doi.org/10.1071/fp04063
Romanzetti S, Halse M, Kaffanke J, Zilles K, Balcom BJ, Shah NJ (2006) A comparison of three SPRITE techniques for the quantitative $3 \mathrm{D}$ imaging of the Na-23 spin density on a $4 \mathrm{~T}$ whole-body machine. J Magn Reson 179:64-72

Schindelin J, Arganda-Carreras I, Frise E, Kaynig V, Longair M, Pietzsch T et al (2012) Fiji: an open-source platform for biological-image analysis. Nat Methods 9:676-682. https://doi.org/10.1038/nmeth.2019

Shokri N, Lehmann P, Or D (2010) Liquid phase continuity and solute concentration dynamics during evaporation from porous media: pore scale processes near vaporization surface. Phys Rev E 81:046308

Simpson MJ, Simpson AJ, Gross D, Spraul M, Kingery WL (2007) H-1 and F-19 nuclear magnetic resonance microimaging of water and chemical distribution in soil columns. Environ Toxicol Chem 26:1340-1348. https://doi. org/10.1897/06-478r.1

Sinha NT, Singh BK (1974) Effect of transpiration rate on salt accumulation around corn roots in a saline soil. Agron J 66: $557-560$

Stingaciu LR, Pohlmeier A, Blümler P, Weihermüller L, Stapf S, Vereecken H (2009) Characterization of unsaturated porous media by high-field and low-field NMR relaxometry. Water Resour Res 45:W08412

Stingaciu LR, Weihermüller L, S. H-P, Stapf S, Vereecken H, Pohlmeier A (2010) Determination of the pore size distribution - a comparison study of laboratory methods. Water Resour Res 46:W11510 doi:https://doi.org/10.1029/2009 WR008686

Van As H (2007) Intact plant MRI for the study of cell water relations, membrane permeability, cell-to-cell and longdistance water transport. J Env Bot 58:743-756

Veliyulin E, Egelandsdal B, Marica F, Balcom BJ (2009) Qunatitative 23Na magnetic Reonance imaging of model foods. J Agric Food Chem 57:4091-4095. https://doi. org/10.1021/jf9000605

Welle PD, Mauter MS (2017) High-resolution model for estimating the economic and policy implications of agricultural soil salinization in California. Environ Res Lett 12:1-17

Publisher's note Springer Nature remains neutral with regard to jurisdictional claims in published maps and institutional affiliations. 